\title{
Diatrizoic Acid
}

National Cancer Institute

\section{Source}

National Cancer Institute. Diatrizoic Acid. NCI Thesaurus. Code C47483.

An org anic, iodinated radiopaque X-ray contrast medium used in diagnostic radiog raphy. The iodine moiety of diatrizoate is not penetrable by X-rays, therefore it blocks the X-ray film exposure by radiation. This makes it possible to distinguish, on X-ray film, body parts that contain diatrizoate meglumine from body parts that do not contain this agent and allows for visualization of different body structures. 